ISSN: 0514-7336

DOI: http://dx.doi.org/10.14201/zephyrus2017803347

\title{
PINTURA ESQUEMÁTICA Y TERRITORIOS DE LA PREHISTORIA RECIENTE EN LA CUENCA INTERIOR DEL TAJO
}

\section{Schematic paintings and territories in the Late Prehistory of inner Tagus basin}

\author{
M. a Ángeles Lancharro Gutiérrez y Primitiva Bueno Ramírez \\ Área de Prehistoria. Dpto. de Historia y Filosofía. Facultad de Filosofía y Letras. Cl Colegios, 2. 28802 Alcalá de \\ Henares.Correo-e: angeles.lancharro@uah.es; p.bueno@uah.es
}

Recepción: 21/03/2017; Revisión: 20/06/2017; Aceptación: 23/07/2017

Resumen: El mapa tradicional del Arte Postpaleolítico de la Península Ibérica dejaba fuera de su geografía una parte importante de las tierras interiores. Ausencias generalizadas de población prehistórica eran la tónica que explicaba la escasez de proyectos de investigación. Afortunadamente, los trabajos de campo de los últimos años han comenzado a mostrar una riqueza demográfica y una simbología totalmente inédita. Un estudio de su visibilidad próxima, de sus posiciones predominantes y de su imbricación en áreas de fuerte implantación desde el Neolítico al Bronce avanzado ofrece, por primera vez, una imagen única de la ocupación sin fisuras de todas las sierras interiores con sus correspondientes áreas de valle. Símbolos con gran arraigo en todo el arte esquemático clásico protagonizan los paneles de la zona e, incluso, figuras de mayor antigüedad apuntan secuencias gráficas de profundo recorrido.

Palabras clave: Península Ibérica; territorios interiores; Holoceno; simbología; análisis SIG.

Aвstract: The traditional map of Postpaleolithic Art in the Iberian Peninsula left out a significant part of its inner territories. Their presumed lack of population justified the absence of research projects in the region. However, fieldwork done in the last few years in Neolithic, Megalithic and Chalcolithic settings within this area has suggested demographic abundance and an entirely unknown symbology. An analysis of their near visibility, their prominent position, and their overlapping with areas inhabited from the Neolithic to the late Bronze Age, reveals for the first time the continuing settlement of all the inner ranges and valleys of these territories.

Common symbols in classical schematic art play a leading role in the area's panels. In addition, the oldest figures point to the existence of long graphic sequences.

Key words: Iberian Peninsula; inner territories; Holocene; simbology; Gis spatial analysis.

\section{Introducción}

La generación de estrategias de investigación debe apoyarse en preguntas que amplíen las posibilidades de estudio. En el caso de la Prehistoria Reciente, nuestro grupo ha insistido en el valor de los yacimientos decorados como materialización no sólo de ocupaciones humanas, sino de los modos de uso y circulación de los territorios que estas ocupaban. La experiencia adquirida en otros entornos peninsulares del interior y del suroeste nos permite asumir como punto de partida un modelo de 
integración de yacimientos decorados y no decorados que tiene un peso contrastado. Las sierras como ámbito económico de buenos pastos, fuentes de agua o lugares extractivos forman parte de las topografías de las ocupaciones humanas que se sitúan en pie de sierra o en valle para desarrollar modos de vida agropecuarios, y que tienen otro de sus puntos de referencia en las líneas de agua. Unos y otros yacimientos están conectados como parte de un entramado cultural compacto que asegura el tránsito $\mathrm{y}$ uso de diferentes nichos económicos de recorrido vertical y horizontal. Los marcadores gráficos se constituyen en visi-

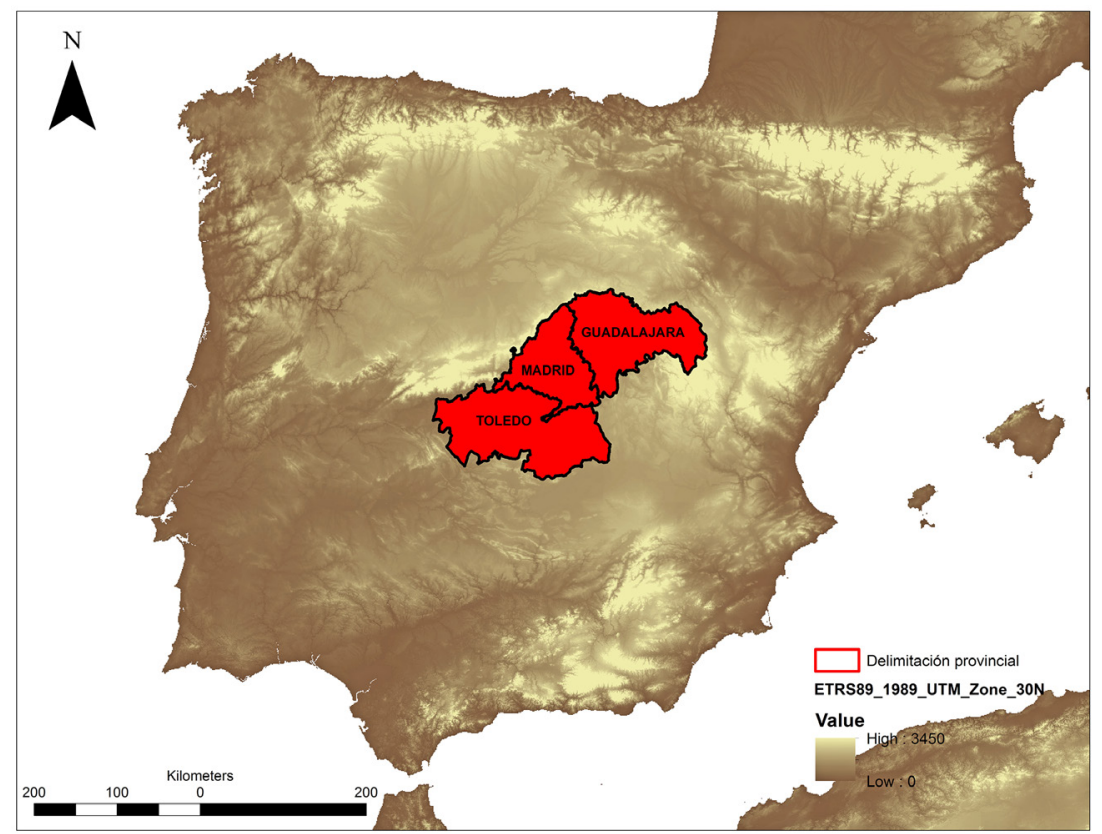

FIG. 1. Mapa peninsular delimitando las provincias interiores; cartografia a partir de ASTER, Global Digital Model (http://asterweb.jpl.nasa.gov/). grupos prehistóricos y son un excelente punto de partida para plantearse prospecciones intensivas en sus territorios próximos.

El Área de Prehistoria de la Universidad de Alcalá de Henares (UAH) ha venido desarrollando proyectos de investigación en relación con los sistemas de ocupación en la Prehistoria Reciente del Tajo Interior ${ }^{1}$. En todos ellos el estudio de los marcadores gráficos ha formado parte ineludible de las propuestas interpretativas sobre estos grupos. El background teórico y metodológico de estos

1 La tesis doctoral Grafias y territorios megaliticos en la cuenca interior del Tajo: Madrid, Toledo y Guadalajara se ha realizado con el apoyo de una beca FPI en el Área de Prehistoria de la Univ. de Alcalá de Henares. Este trabajo se integra en el conjunto de resultados del proyecto HAR2012-34709 actualmente en vigor. La colaboración de B. Maquedano y J. R. Villa, del Servicio de Arqueología de la Consejería de Educación y Cultura de la JCLM, ha sido básica en la consulta de cartas arqueológicas. El apoyo de la técnico de arqueología de Castilla-La Mancha, en Guadalajara, T. Sagardoy, ha sido fundamental, así como la información proporcionada por los agentes medioambientales D. Moreno, J. Á. Jambrina, E. Fuertes y F. Bravo. trabajos es el punto de partida de un análisis más amplio, parte de cuyos resultados exponemos aquí (Bueno et al., 2004, 2006, 2008, 2009a, b).

Ofrecer una imagen actualizada de los marcadores gráficos del sector pasa por la inserción de todos los datos publicados sobre soportes decorados de distinto tipo en las actuales provincias de Toledo, Madrid y Guadalajara, y su georreferenciación cartográfica (Fig. 1). La aplicación de tecnologías SIG (Sistemas de Información Geográfica) y sus herramientas de análisis nos permite estudiar las relaciones entre este tipo de yacimientos y otras huellas de actividad humana en la región, incluyendo no sólo áreas de habitación y áreas funerarias, sino evidencias extractivas. La imagen conseguida ofrece, por primera vez en los registros del Arte esquemático peninsular, una referencia sólida sobre el papel de los marcadores gráficos en estos territorios interiores, como algunos trabajos previos señalaban (Lancharro, 2012).

El valor de lo obtenido aporta un argumento más a las evidencias de una paleodemografía consolidada en las ocupaciones agropecuarias y metalúrgicas del área, apuntando incluso a fases más 
antiguas que pueden relacionarse con el Paleolítico y el Epipaleolítico.

\section{Metodología. Variables locacionales (SIG) y modelos de integración de yacimientos decorados y no decorados}

Esta propuesta necesita de argumentos que herramientas como los Sistemas de Información Geográfica (sIG) proporcionan. Aspectos como la visibilidad (Cruz, 2004; Fairén, 2002; Lake y Woodman, 1998; Martínez Bea, 2006; Wheatley y Gillings, 2002; Zamora, 2006) o invisibilidad (Gillings, 2015), intervisibilidad (Gafnney y Stânçic, 1991; Fisher et al., 1997; Wheatley, 1995, 1996; Lake et al., 1998), exposición visual (Llobera, 2003), visibilidad acumulada y total (García Sanjuán et al., 2006; Llobera et al., 2010) muestran su potencial, del mismo modo que los relacionados con la red de comunicaciones y los accesos al lugar (Fairén, 2004; Gibson, 2007; Martínez y Martorell, 2011; Murrieta, 2012; Van Leusen, 1999). Hemos elegido como criterio de análisis la capacidad de los abrigos decorados de controlar visualmente y articular, desde su posición estratégica, el territorio circundante.

Las metodologías desarrolladas en la urbanística y la paisajística han influido en ocasiones positivamente en los análisis arqueológicos (Wheatley y Gillings, 2000). T. Higuchi (1983), en su estudio del paisaje fuera del ámbito urbano, trataba la estructuración del campo visual desde cualquier punto de observación, elaborando para ello un índice con ocho criterios que son la causa de su variabilidad. Esta teoría ha tenido impacto en Arqueología y es la base de la descomposición del modelo binario, visible o no visible, y su categorización en niveles diferenciados por su 'bondad visual'. Entre los principios sustanciales en esa correcta captación sensorial del paisaje se halla la distancia, que desde el punto de observación establece la clasificación de lo que vemos o percibimos:

1. Distancia inmediata, en la que se pueden ver los árboles individualmente con hojas, ramas y troncos. El observador relaciona el tamaño del árbol en proporción con otros y puede establecer comparaciones; entrarían en juego adjetivando el conjunto sentidos como el del olfato o el oído. Este rango respondería a 60 veces el tamaño del objeto que toma de referencia: para Higuchi es el árbol en torno al que desarrolla su transformación sensorial.

2. Distancia media, donde se perfilan las copas, la textura, los grupos de árboles y ciertas características del paisaje («we see forest rather than the trees»), mientras que otros sentidos (olfato, oído...) matizan su efecto. Este intervalo es parte principal de la escena, desplegando sensación de profundidad. Comienzan a influir aquí los elementos atmosféricos que alterarían lo percibido, como la neblina, la bruma, etc. Caracteriza todo esto una distancia de hasta 1.100 veces el tamaño del objeto de referencia.

3. Por último, donde finaliza el tramo anterior se establece una zona en la que percibimos el ambiente boscoso con cambios en la tonalidad e iluminación, dando la apariencia en conjunto de un telón de fondo. Son más ostensibles las unidades topográficas: valles, crestas y otras peculiares. Las condiciones atmosféricas afectan especialmente a este escenario (Higuchi, 1983: 12-16).

El método es aplicable en otros campos modificando el objeto que nos sirve de referencia (Wheatley y Gillings, 2000; Ogburn, 2006). En el estudio del paisaje prehistórico en la cuenca del Tajo, a su paso por el área extremeña, se analiza con este método el abrigo decorado del arroyo Estanque (Cerrillo, 2011).

En nuestro caso el objeto de referencia es 1,7 m, que tomamos como altura media de un individuo erguido, si bien alguna variación a una menor no altera el análisis, como hemos comprobado in situ sobre el pequeño llano circundante a los paneles en el abrigo de Los Casares iII. Según esta medida y la teoría de Higuchi, las distancias arrojan un resultado de un tramo de $102 \mathrm{~m}$ desde el punto de observación que goza de una óptima visibilidad, un segundo tramo entre los 102 y 1870 m que está caracterizado por una buena visibilidad, distancia esta última a partir de la cual se extiende el tercer tramo que calificamos de baja visibilidad. Cada uno con los rasgos y cambios sensoriales descritos en los puntos anteriores. Los condicionantes medioambientales, las oscilaciones en la transparencia del aire 
y sin duda el carácter del objetivo a visualizar especifican el proceso y los resultados (Zamora, 2006; Wheatley, 2014).

Tradicionalmente, la posición de los abrigos decorados se define por su prominencia y elevada altitud, rasgo asociado a la capacidad de observación sobre el territorio. Adoptando la referencia de los valores de la cuenca visual en rangos, tomamos un radio de $2 \mathrm{~km}$ en torno al yacimiento para calcular el índice de altitud relativa (AR) del abrigo, utilizando para ello el algoritmo aplicado en el estudio del paisaje castreńo de la Edad del Hierro en el No peninsular (Parcero, 2002: 69-70), que asigna dos radios de 800 y 2000 m desde el yacimiento, con el objeto de extraer sendos patrones de localización. La medida de $2 \mathrm{~km}$ en el abrigo de Los Casares III se corresponde con los tramos $1 .^{\circ}$ y $2 .^{\circ}$, que coinciden muy aproximados con la distancia en la que se distinguen elementos, objetos o signos de actividad y a la inversa, las pinturas y la formación rocosa que las sustenta.

Abordamos el análisis de los abrigos rupestres postpaleolíticos a partir de la estructuración de la cuenca visual bajo criterios que parten de estos presupuestos metodológicos y, con sus resultados, establecemos una aproximación al espacio y el territorio que comparten y definen, junto con las propias grafías (grabados, pinturas y piezas exentas).

La cartografía utilizada proviene del Ministerio de Fomento, a partir de sus institutos y centro de descargas (IGN, CNIG), visores (IBERPIX) y proyectos coordinados por este organismo (sIOSE). Desde el Centro de Descargas y catálogo de productos del CNIG, obtuvimos las cuadrículas correspondientes del Modelo Digital del Terreno MDTo 5/MDTO5-LIDAR procedente del Plan Nacional de Ortofotografía Aérea (PNOA), que resulta de la interpolación de modelos digitales del terreno de $5 \mathrm{~m}$ de paso de malla, con la misma distribución de hojas del MTN. Estos nos permiten extrapolar modelos muy ceñidos a la topografía real del terreno y escenificar con propiedad el contexto del abrigo decorado y sus relaciones con otros yacimientos de diferentes esferas: económicas y sociales (poblacionales y funerarias) y demás marcadores gráficos.

\section{Un caso de estudio en los territorios del interior peninsular: visibilidad, localización geoestratégica y relaciones culturales en Sierra de Caldereros, Guadalajara}

Proponer un caso de estudio es el modo más sencillo de hacer patentes los objetivos, metodología y resultados del protocolo que hemos explicado. Para ello hemos seleccionado los datos de los abrigos de la Sierra de Caldereros, en Guadalajara, partiendo de la posición y contenido del situado en la zona conectada con el valle, el abrigo de Los Casares III. La información recogida de este abrigo y el resto del conjunto parte de las cartas arqueológicas consultadas, además de la que se obtuvo de la visita a los yacimientos. Desgraciadamente, muchos de los abrigos citados en este texto no disponen de información publicada, más allá de la referencia sintética que se recoge en la Carta Arqueológica de la Junta de Castilla-La Mancha.

Nuestra intención no se dirige al estudio gráfico-descriptivo del yacimiento, sino a tomar este como punto de partida para un análisis que permita hacer visible la estrecha relación entre las ubicaciones conectadas con explotaciones agropecuarias y las relacionadas con las zonas altas, en el sentido que hemos argumentado en otras ocasiones.

La Sierra de Caldereros se encuentra en la zona oriental del Sistema Ibérico. Es un paraje natural declarado, cuya figura se erige sobre los valles del señorío de Molina, tradicional territorio con accesos al Ebro. Algunas de las características geológicas de esta área concuerdan con las de conjuntos esquemáticos conquenses. Ese es el caso del abrigo mencionado en el que destaca un panel lineal con antropomorfos integrables en el conjunto de los ancoriformes y oculados, y una figura de carácter naturalista. La bicromía de las figuras, rojo de relleno y puntos en blanco de contorno, remite a ejemplos del arte esquemático clásico (Breuil, 1933), y abre posibilidades de relación con referencias detectadas al interior de los megalitos. La asociación de un panel de grabados en posición cenital colabora en transmitir una imagen compleja de este yacimiento. Evidencias singulares que, junto con la posición del 


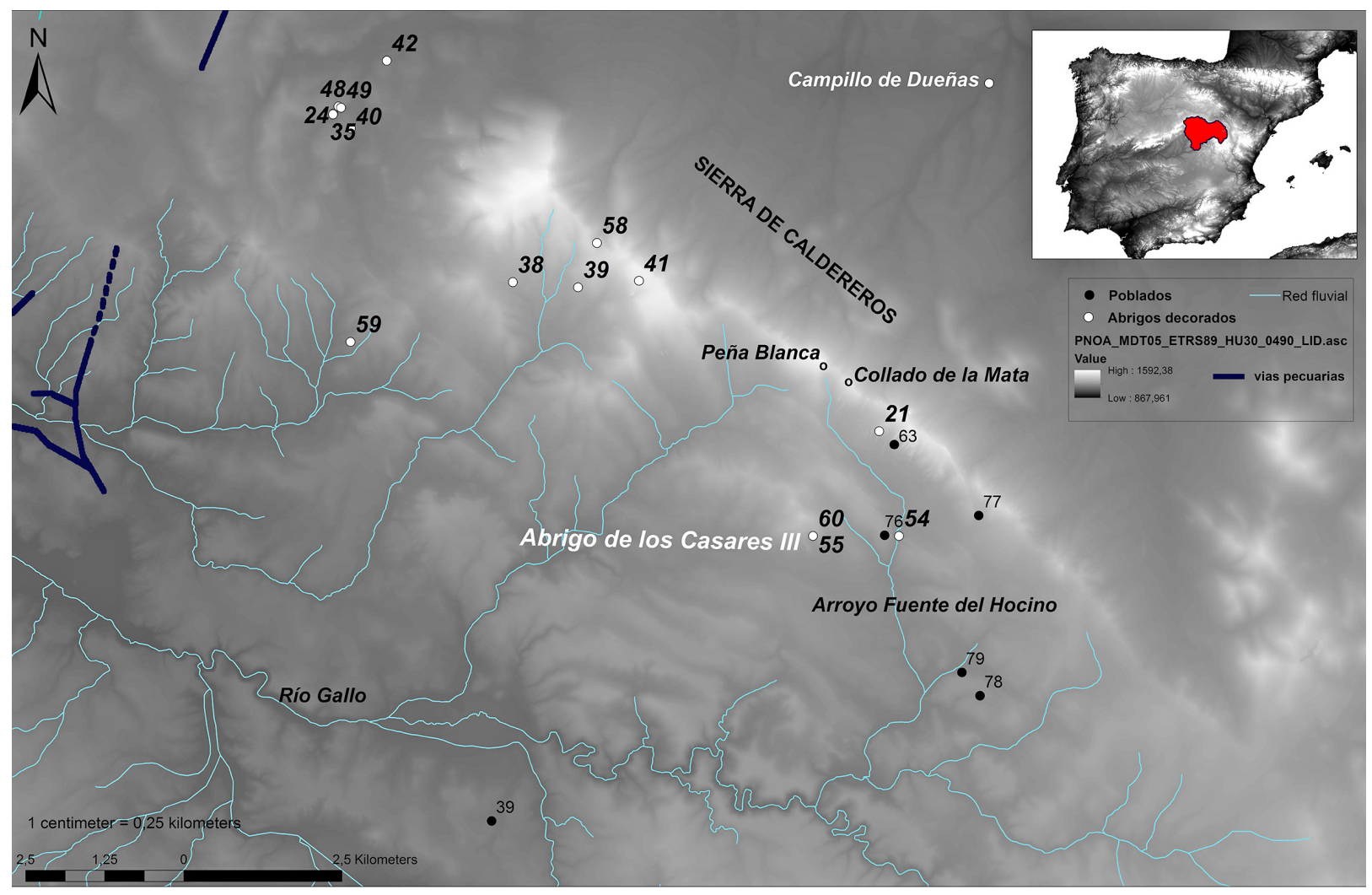

FIG. 2. Unidad geográfica de la Sierra de Caldereros, poblados, abrigo de Los Casares III y resto de marcadores gráficos numerados en Arte Esquemático en el Tajo (AETA): 21) abrigos de Zafra I-VIII; 24) Peñahita I; 35) Peñahita II; 38) grabados de Cerrada de Prado; 39) grabados de Coverteruela; 40) cazoletas y grabados de Peñahita; 41) grabados de Castilmayor; 42) cazoletas de las Majoneras; 48) Peñahita III; 49) Peñahita IV; 54) abrigos del Hocino I-IV; 55) y 60) abrigo y grabados de Los Casares III; 58) menhires de Coverteruela; 59) abrigo del Picozo. Poblados: 63) Zafra; 76) Prado de Alcalá; 77) Corral Viejo; 78) Los Costerones; 79) La Loma; 39) El Aulladero (WMS de vías pecuarias MAGRAMA y cartografía del CNIG).

sector en relación a importantes conjuntos levantinos, son las que nos inclinaron a elegir este caso.

La unidad geográfica en la que se enmarca dispone de otros soportes pintados: los abrigos del Hocino, los abrigos del Castillo de Zafra, el del Picozo o los de Peñahíta, todos con Arte Esquemático, acompañado de evidencias naturalistas del denominado estilo levantino. Algunas de las figuras -zoomorfas y antropomorfas-, entrarían sin dificultad en los que caracterizamos como estilo v. La mayor acumulación, pese a no disponer aún de un estudio exhaustivo, es la de los abrigos de Zafra que se asocian a la prominencia topográfica más identificativa de este ámbito territorial.

En el interior y serranía a mayor altura, se salpican por la geografía rocas grabadas al aire libre con cazoletas y líneas, y dos menhires. Los restos de habitación, por el momento, se hallan en la vertiente $s$ de la sierra y cercanos a varios de los abrigos, en el poblado del Castillo de Zafra de adscripción neolítica-calcolítica en las inmediaciones de los abrigos de Zafra; el de Prado de Alcalá, un yacimiento del Bronce que conserva estructuras de muros ciclópeos y restos de cerámica a mano (Carta Arqueológica); el poblado de Corral Viejo, también del Bronce, se trata de un asentamiento de grandes dimensiones con estructuras de mampostería en seco en las terrazas escalonadas, debido al lugar que ocupa de cárcava estrecha que desemboca en el valle de la Dehesa de Zafra (Carta Arqueológica); dos yacimientos más se ubican al s del municipio de Hombrados y sobre una zona amesetada denominada el Saucejo, el de 


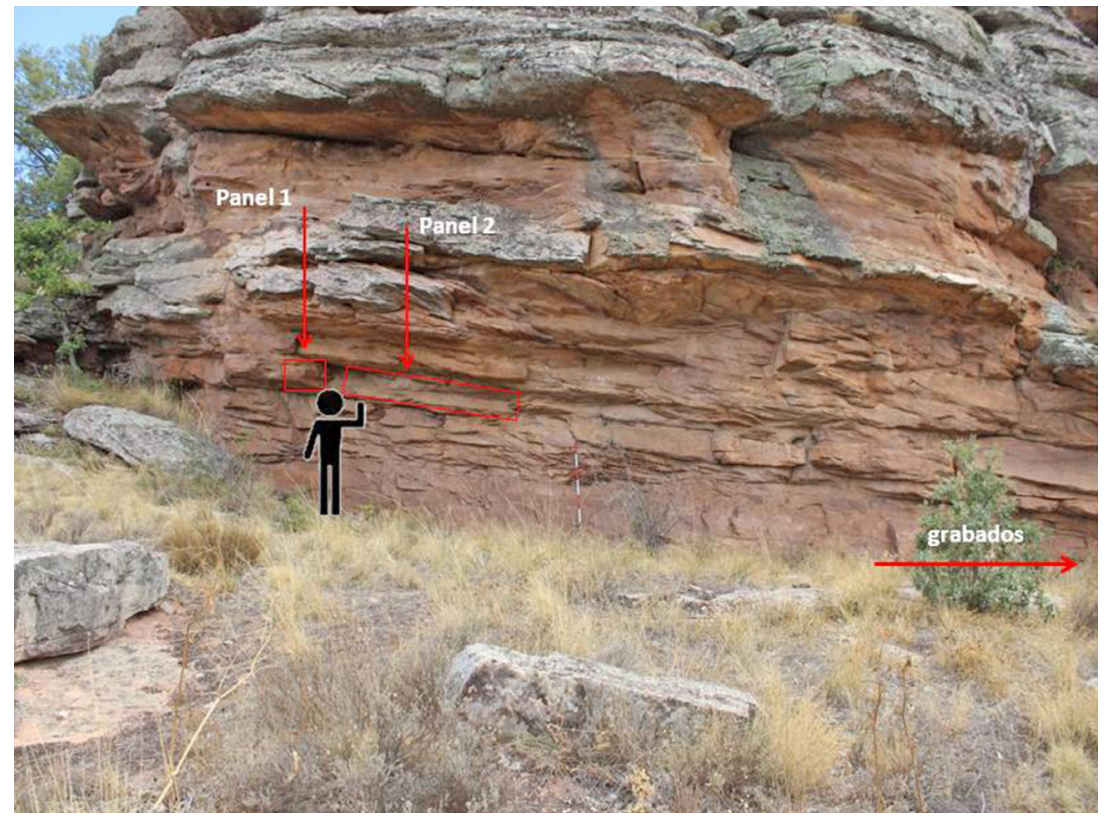

FIG. 3. Perspectiva desde el o hacia el abrigo de Los Casares III.

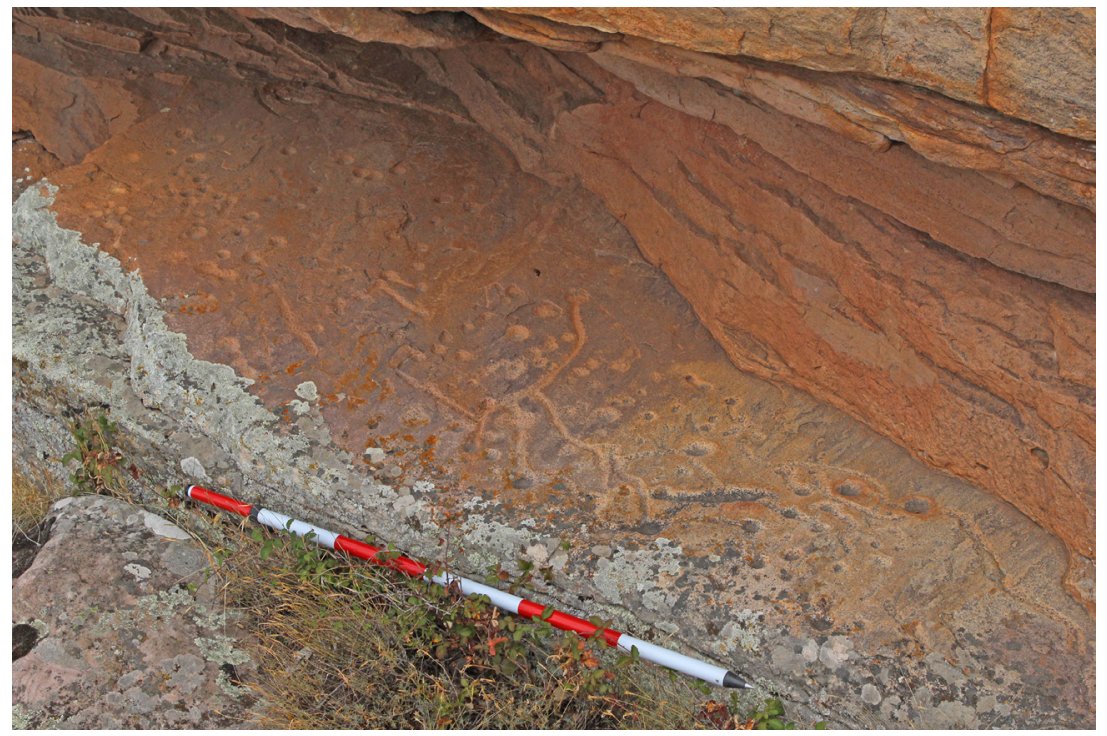

FIG. 4. Grabados próximos a los paneles pintados sobre una roca en posición cenital.

calcolítica; y en Torrecuadrada (Torrecuadrada de Molina) restos de hábitat con cerámica campaniforme (Bueno et al., 1995) (Fig. 2).

En relación a los caminos tradicionales o cańadas, unos $6 \mathrm{~km}$ al so del yacimiento, pasa por el término de Prados Redondos el cordel que, rodeando Caldereros por su depresión occidental, se dirige hacia el $\mathrm{N}$ en dirección a Tortuera (wMs Magrama de Vías pecuarias). El espacio comprendido entre los yacimientos de $\mathrm{Za}$ fra, El Hocino y Casares III es un paso natural a esta sierra por el barranco del Hocino y la vega a través de los valles; la senda continúa girando a la izquierda hacia Campillo de Dueñas, discurriendo entre los picos de Peña Blanca y Collado de la Mata, en dirección $\mathrm{N}$. Si bien la sierra carece de caminos señalados en dichas vías pecuarias, es factible que la ruta y el paso por el que se accede facilitasen el movimiento.

El abrigo de Los Casares III se sitúa en la vertiente meridional de un destacado machón de arenisca, cuya parte más alta tiene ocupación protohistórica e histórica. Los paneles pintados (2) no resultan muy visibles al incluirse en una estrecha franja del tercio medio del sopor-

La Loma, un poblado calcolítico con restos líticos y cerámicos, y Los Costerones, a $250 \mathrm{~m}$ al sE del anterior, en el que se halló material lítico (sílex) que hace pensar en un taller (Carta Arqueológica). A 8 $\mathrm{km}$ hacia el $\mathrm{s}$ de Los Casares III, tiene lugar otro tipo de actividad del que nos resta nuevamente, industria lítica tallada, en el sitio del Aulladero (Prados Redondos) posiblemente de cronología neolítica y te, protegidos por una visera. En la parte baja del abrigo y en su extremo o se observa un conjunto de grabados de cazoletas y líneas con orientación cenital sobre el soporte rocoso natural. La orientación del abrigo es la contraria a la mantenida por la ocupación reciente de la zona superior. El terreno inmediato tiene pendientes muy moderadas y amplia visibilidad en la misma dirección (Figs. 3 y 4). 


\section{Caminando entre sierras y valles}

Caldereros es un territorio poco alterado por infraestructuras, por lo que los aspectos del campo de visión no se ven afectados en esencia. Como decíamos, es un accidente geográfico de fuerte impacto visual desde los territorios circundantes, tanto por sus formaciones de arenisca en moles, como por su color rojizo y su aspecto estratificado.

La orientación del abrigo hacia el o da lugar a una cuenca visual en ángulo muy abierto de casi $180^{\circ}$ en un abanico O-SE, alargándose sobre unos $20 \mathrm{~km}$ aproximadamente. El tramo de visibilidad óptima (aprox. $102 \mathrm{~m}$ ) inmediata al abrigo afecta a la zona so en especial y el segundo tramo (aprox. 102-1870 m) incide en las áreas de cultivo y ganaderas adyacentes al abrigo en un perímetro semicircular O-S-E. Son estos primeros tramos los que acumulan mayor exposición visual, mientras que el último tramo a partir de $2 \mathrm{~km}$ sufre una mayor fragmentación, si bien tiene una proyección más allá de los $20 \mathrm{~km}$, fuera de los márgenes de nuestro MDT, que indica, como apuntábamos, la percepción de la Sierra de Caldereros y sus formaciones surorientales desde una larga distancia (Fig. 5).

De ello se deduce que la formación resulta más visible que el propio abrigo, lo que adjudica a la topografía natural un papel que otros autores han señalado (Bradley et al., 1994; Martínez, 1998).

En la situación actual de nuestros conocimientos, Los Casares III y los abrigos más cercanos, como el Hocino I-Iv, Zafra I-VıII, señalizan la entrada a la sierra, organizando con sus campos visuales espacios más pequeños. La sugerente concatenación y complementación de cuencas visuales es un hecho. Los Casares III se orienta hacia la vertiente s sobre

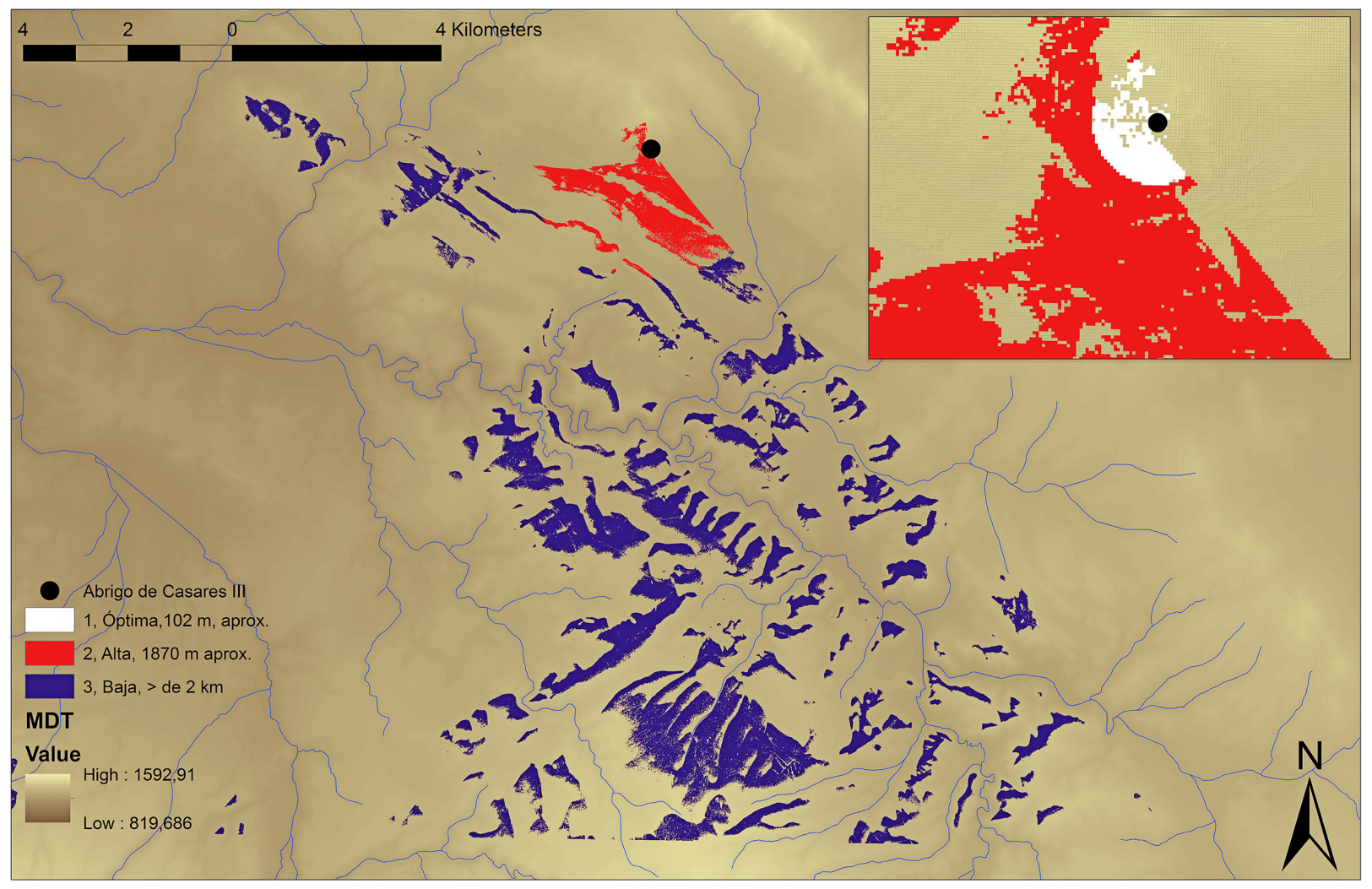

FIG. 5. Articulación de la cuenca de Los Casares III en los tres niveles de distancia visual elaborados a partir de la propuesta de Higuchi: distancia de visibilidad óptima $(102 \mathrm{~m})$, buena $(102-1870 \mathrm{~m})$ y baja $(>1870 \mathrm{~m})$; arriba a la derecha detalle del primer tramo de $102 \mathrm{~m}$, inapreciable en el mapa central (fuente cartográfica: $C N I G$ ). 


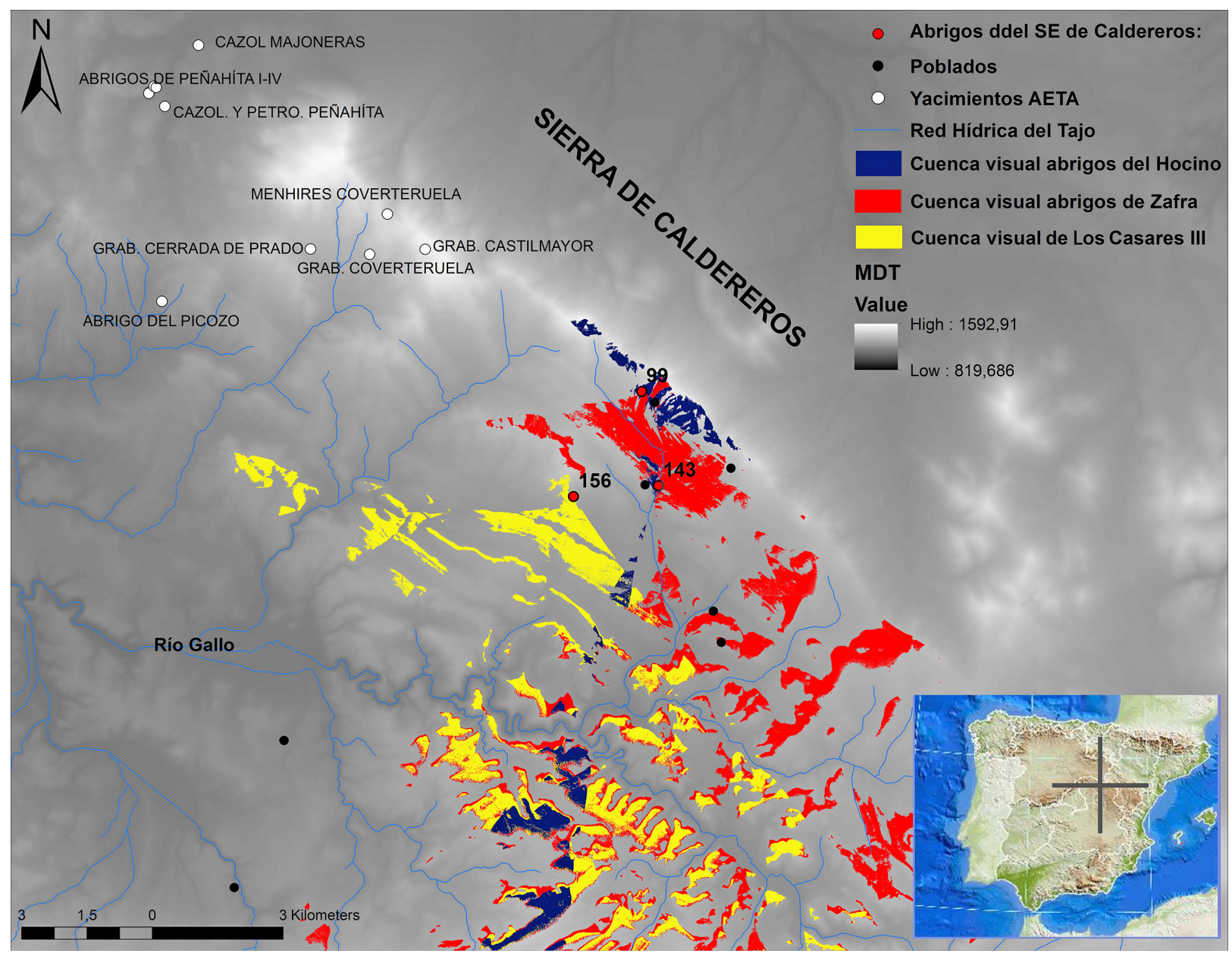

Fig. 6. Cuencas de visibilidad de los tres abrigos del SE de Caldereros: 156) Los Casares III; 143) El Hocino I-IV; 99) Zafra I-VIII. Distribución de poblados y otros marcadores gráficos recogidos en la BD del Arte Esquemático en el interior del Tajo (AETA) (fuente cartográfica: $C N I G$ ).

las llanuras que se extienden a sus pies, donde aparecen los lugares de hábitat; los abrigos del Hocino mantienen nexos de visibilidad hacia el barranco que da acceso a un segundo valle hacia el interior $y$ dirección N, y los de Zafra dirigen su campo visual hacia este último valle y los accesos a las cumbres de Caldereros, donde se distribuyen grabados y menhires, además de hábitats próximos. Un territorio en el que los símbolos grabados y pintados identifican referencias topográficas y, muy probablemente, modos de uso y tránsito por el mismo (Figs. 6 y 7).

Los temas y técnicas que presenta el conjunto de los sitios referenciados coinciden en repertorios del arte esquemático, un conjunto cuya genérica cronología entre el Neolítico y la Edad del Bronce es comúnmente aceptada. En los abrigos de Zafra, zigzags rellenos en rojo, otros geométricos en negro, figuras humanas en golondrina, en doble $\mathrm{Y}$, figura animal o series de puntuaciones; en el Hocino figuras humanas en cruz, series y agrupaciones de puntos y figuras animales. El uso del blanco, tan escaso por sus problemas de conservación, tiene evidencias también en Zafra, lo que añade otra similitud entre estos yacimientos. Por tanto, un conjunto coherente técnica y temáticamente, incluso en el hecho de que algunas figuras permiten sospechar secuencias gráficas más antiguas. La diferencia más evidente es que Los Casares III alberga antropomorfos 




Fig. 7. Cuenca de visibilidad acumulada (CVA) de los tres abrigos del SE de Caldereros: 156) Los Casares, 143) Abrigos de Hocino, 99) Abrigos de Zafra. La concurrencia visual de los tres se encuentra en zonas que apuntan de s a N la entrada a la sierra a través del Barranco del Hocino (fuente cartográfica: CNIG).

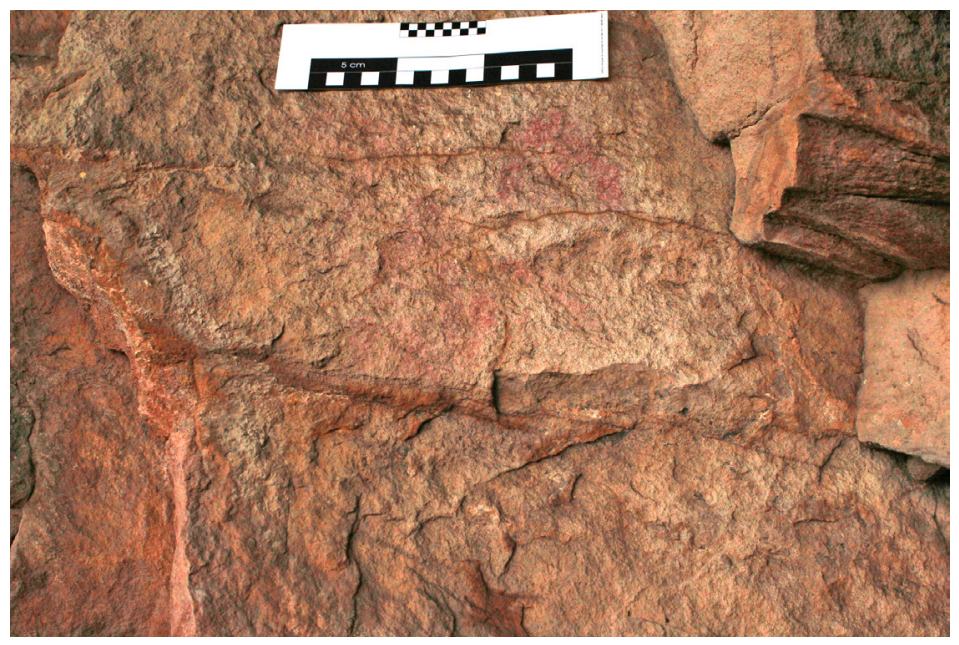

(C) Universidad de Salamanca clasificables en el conjunto de los oculados, dispuestos en sentido horizontal y realizados con una técnica bícroma que los singulariza.

Su organización en línea asocia tres figuras en el panel más pequeño (un posible oculado sencillo, una figura en golondrina y un tema geométrico: ángulo más ondulado) (Fig. 8) y tres oculados en el mayor, con el posible repintado de una figura de jabalí para realizar un cuarto

FIG. 8. Panel 1 en ángulo recto respecto al $n .^{\circ} 2$, con tres figuras humanas. 


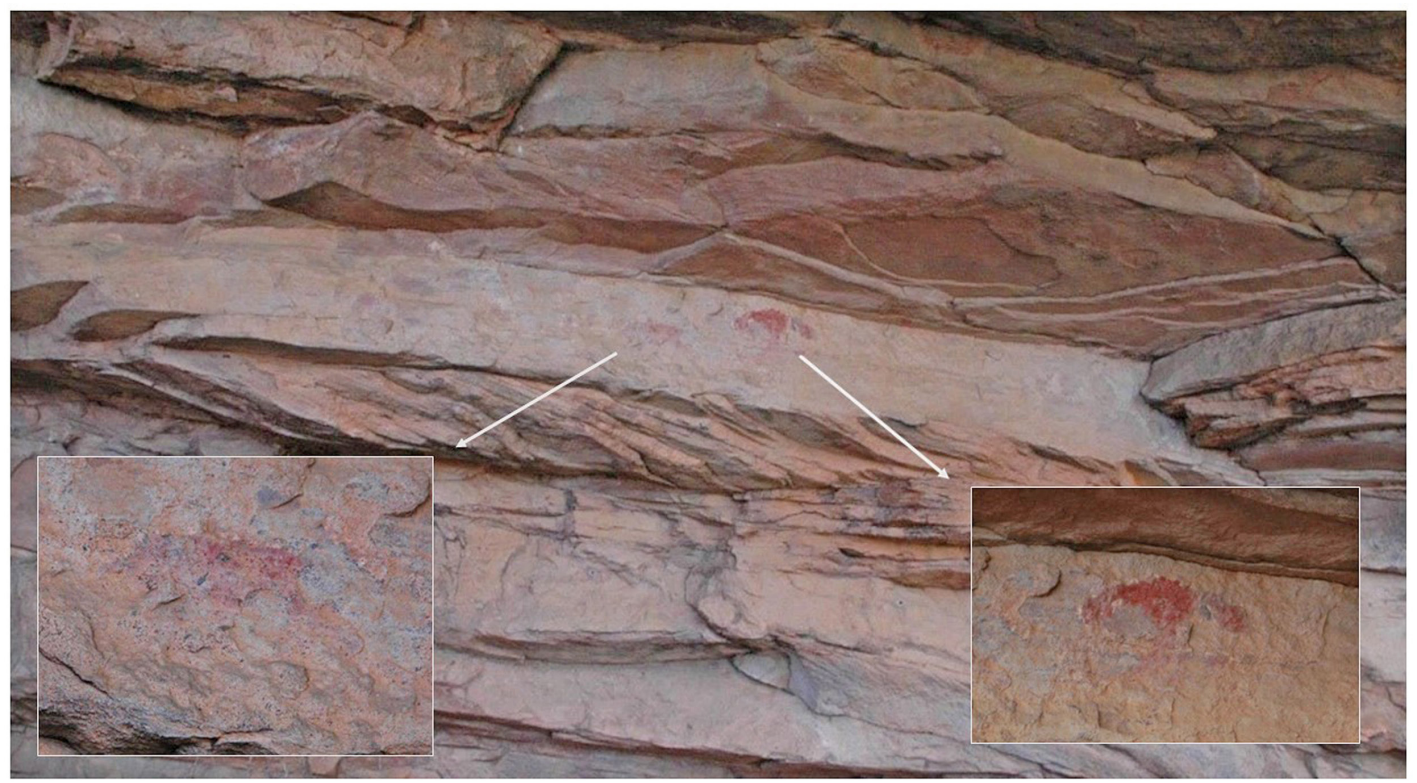

FIG. 9. Panel II en el que se representan 5 motivos bicromos: arriba panel principal de Los Casares III; en centro detalle del oculado central en rojo rodeado de puntos blancos y a la izquierda del jabali sobre el que se ha repintado otro oculado; abajo detalle a la izquierda del segundo oculado, peor conservado pero también circundado de puntos blancos; a derecha detalle del primer oculado.

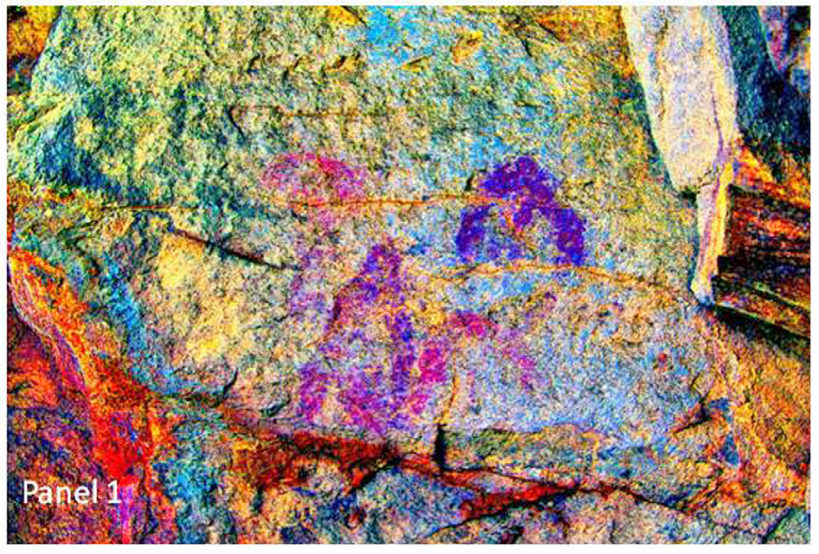

\section{PANEL 1}

Panel con las 3 figuras (espacio de color LDS)

Motivo 1 (espacio de color LDS)

Motivo 2 (espacio de color LRE)

Motivo 3 (espacio de color LDS)
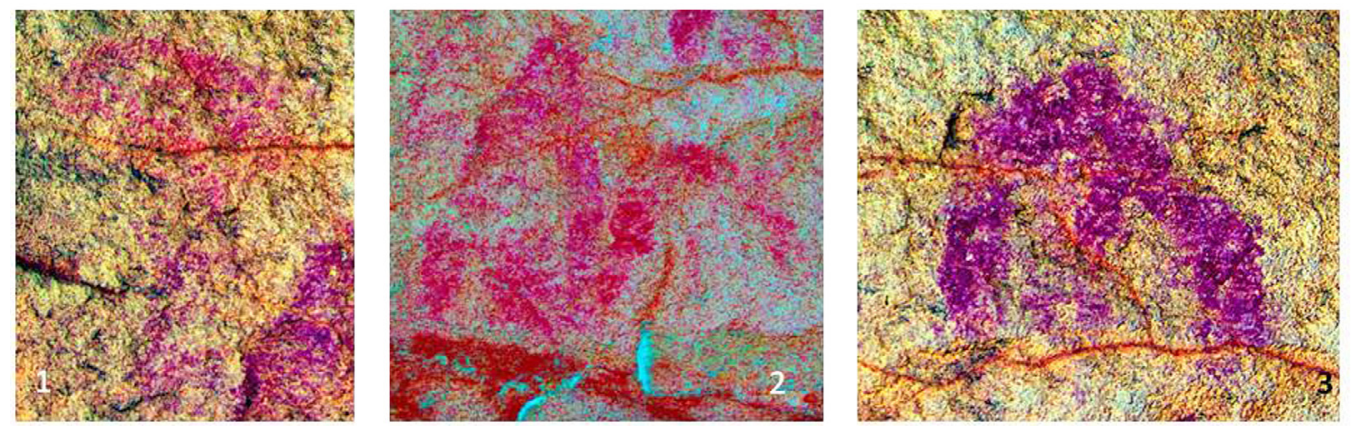

FIG. 10. Desglose de las figuras del Panel 1: posible oculado, figura geométrica y antropomorfo en golondrina; imágenes tratadas con Dstrecht. 

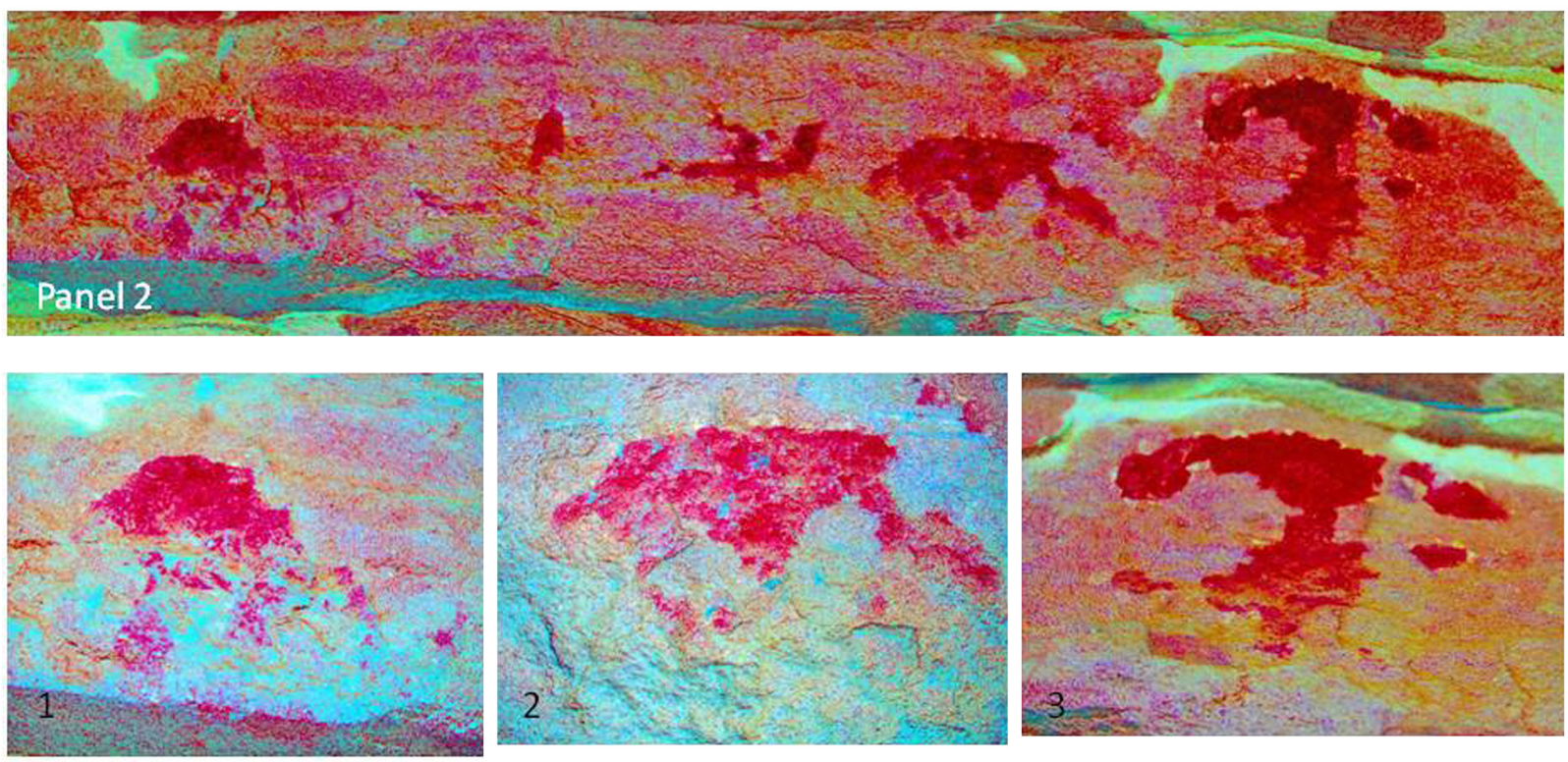

PANEL 2

Panel con las 5 figuras (espacio de color LRE)

Motivo 1 (espacio de color LRE)

Motivo 2 (espacio de color LRE)

Motivo 3 (espacio de color LRE)

Motivo 4 (espacio de color LRE)

Motivo 5 (espacio de color CRGB)


Fig. 11. Desglose de las figuras del Panel 2: imagen superior del panel completo y las siguientes con las figuras; imágenes tratadas con Dstrecht.

motivo oculado. Hay más restos de rojo que pueden indicar un desarrollo más complejo de la superficie originalmente decorada, quizás un antropomorfo con brazos abiertos y restos de otro (Fig. 9).

La documentación gráfica que adjuntamos solo pretende ofrecer una imagen del nivel de conservación de este conjunto. De ahí que hayamos utilizado la sencilla herramienta Dstretch para facilitar una lectura rápida (Figs. 10 y 11).

\section{Balance de resultados}

Los Casares inI es un sitio decorado relacionado con las llanuras que se extienden a sus pies. Sus pequeños paneles no son susceptibles de una visibilidad a larga distancia, comparable a la del machón de arenisca en el que se albergan, manifestando una dicotomía visibilidad del panel versus visibilidad de la referencia topográfica, que abre interesantes reflexiones. Su posición al pie del machón de arenisca otorga una capacidad espacial importante para congregar un numeroso grupo humano. Este aspecto de espacios proyectados presididos por abrigos pequeños debería considerarse en los estudios dirigidos a los yacimientos decorados, pues añade un referente de análisis incuestionable para valorar la "audiencia" de algunos abrigos con arte rupestre (Bradley, 2009). De este modo, las visibilidades menores podrían quedar compensadas por espacios ante los abrigos de importante capacidad de agregación.

La singularidad de las grafías de Los Casares III lo destaca en el conjunto de abrigos de la Sierra de Caldereros. Si bien no disponemos de fechas exactas 
para su realización, sí podemos referenciar que a uno y otro lado del Sistema Ibérico disponemos de datos para enmarcar este tipo de temas. Por un lado, los oculados más elaborados descritos en las sierras conquenses, y recientemente datados en el III milenio cal BC a partir de costras de oxalatos (Ruiz et al., 2012 ); por otro, la existencia de abrigos con oculados en versiones variadas que usan los colores rojo y blanco para su realización (Guillem y Martínez, 2013: 207). El abrigo del Castell de Villafamés es un caso de enorme interés al presentar un conjunto de soluciones gráficas, normalmente enmarcadas en oquedades circulares del mismo estilo que las detectadas en los oculados de los abrigos de Peñahíta actualmente en estudio o de los Forestales (Oliver $e t$ al., 2015). Por tanto, señalando la potencia de referencias adscribibles a un Calcolítico clásico en una zona interior a la que se le otorgaba un papel muy marginal en las ocupaciones con esta cronología.

Las escasas referencias materiales recogidas en la planimetría que adjuntamos conectan sin dificultad el posible hábitat de Torrecuadrada con las extracciones de sílex del Aulladero y la explotación agropecuaria del valle en el que se sitúan. Una cronología del iII milenio para la instalación de la mayor parte de las grafías de Los Casares III, quizás superpuestas a una ocupación más antigua como indica el repintado de una figura de jabalí transformada en ancoriforme, resulta convincente. Secuencias largas con bases epipaleolíticas o incluso paleolíticas parecen desprenderse de algunos de los temas identificados en los abrigos de la sierra.

La relación de estas figuras con un panel cenital, en el suelo del abrigo, recuerda de nuevo algunas posiciones detectadas en el arte esquemático ibérico. Por un lado, las conocidas en abrigos en el entorno de poblaciones neolíticas y calcolíticas extremeñas, el poblado de Los Barruecos es un buen caso de estudio (Cerrillo, 2006; Sauceda, 2001). Por otro, las rocas grabadas del mismo modo en los contornos de poblados de la prehistoria reciente del área conquense. La más que convincente contemporaneidad de pinturas y grabados concuerda con los contextos en que ambas expresiones se detectan. La posición de Los Casares III en el borde externo de

(C) Universidad de Salamanca un machón con ocupación humana se reitera en los abrigos del Hocino que se disponen al pie de otro machón de arenisca con el interés de que se asocian a un poblado de la Edad del Bronce. No tenemos evidencias de ocupaciones del III milenio en Los Casares III, pero la repetición de posición con un enclave tan próximo resulta enormemente sugerente para relacionar estos sitios con un sistema de marcación de delimitaciones de poblados, como propusimos para algunos yacimientos neolíticos y calcolíticos del interior (Bueno et al., 1998).

En su conjunto, los marcadores gráficos en la Sierra de Caldereros son nudos de una red que conecta y comunica la sierra desde su cresta hacia las vertientes $\mathrm{N}$, s y o. La evidente conexión entre las áreas de visibilidad de cada uno de ellos y su coincidencia cronológica, al menos en el Neolítico Final-Calcolítico, permite asegurar que los abrigos constituyen un entramado de marcadores simbólicos que organizan este territorio, asegurando el control de las zonas fértiles de valle y el uso de los recursos de sierra, de un modo perfectamente estructurado. Podemos sospechar que estas redes son de origen antiguo, a tenor de algunas figuras, pero, sin duda, desde el IV milenio en adelante parecen el sistema más extendido de definir territorios.

Hasta donde conocemos, se sitúan siguiendo el modelo de asociación entre marcadores gráficos y áreas de habitación y explotación de recursos. Somos conscientes de que nos faltan contextos funerarios que en otros sectores de la región aparecen en dólmenes o en cuevas naturales. Los menhires citados nos permiten albergar esperanzas en futuras localizaciones de conjuntos megalíticos como el del Portillo de las Cortes cuyo estudio hemos retomado (Bueno et al., 2016). La diferencia altitudinal entre unos yacimientos y otros no es fuerte, dados los 1300 msnm de los yacimientos de Zafra, 1250 msnm de Los Casares III, para bajar a los 1100 de los poblados en Prados Redondos (Fig. 12).

Los símbolos asociados al apogeo del arte esquemático (Martínez, 2006) poseen una representatividad inédita en las regiones interiores, constituyéndose los documentados en las Sierras de Guadalajara en referencias ineludibles para valorar las posiciones 


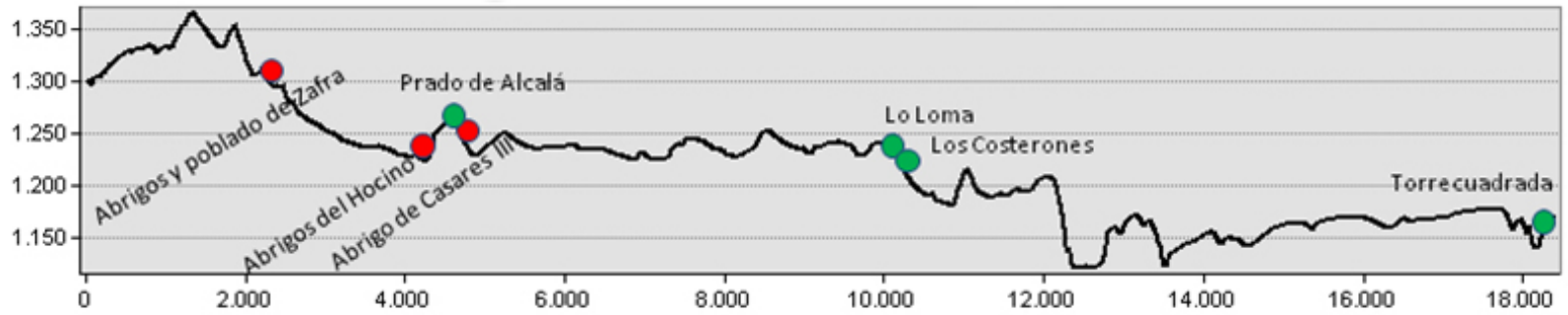

Dirección N-S

Fig. 12. Perfil de elevaciones de Caldereros y algunos de sus yacimientos.

de estos grupos y su capacidad de interacción con el S, SE, o y n de la Península Ibérica. Teniendo en cuenta que las cerámicas campaniformes son la más clara evidencia de redes muy estructuradas, la presencia de estas en los sitios citados, especialmente Torrecuadrada, permite esperar contextos arqueológicos del nivel de los que otros sectores de la Meseta Sur están aportando en los últimos años. La presencia en estas cerámicas de motivos oculados se enriquece constantemente, confirmando el papel de los repertorios gráficos del III milenio cal $\mathrm{BC}$ en la construcción ideológica de la organización social de los grupos de la Prehistoria Reciente del interior peninsular.

\section{Bibliografía}

Bradley, R. (2009): Image and audience: rethinking prehistoric art. Oxford: oup.

Bradley, R.; Criado, R. y Fábregas, R. (1994): "Rock Art research as landscape archaeology: a pilot study in Galicia, northwest in Spain", World Archaeology, 25 (3), pp. 370-390.

Breuil, H. (1933): Les peintures rupestres schématiques dans la Péninsule Ibérique. Paris: Lagny edit., vol. I.

Bueno, P.; Barroso, R.; De Balbín, R. y Carrera, F. (2006): Megalitos y marcadores gráficos en el Tajo Internacional: Santiago de Alcántara (Cáceres). Santiago de Alcántara: Ayto. Santiago de Alcántara.

Bueno, P.; Barroso, R.; De Balbín, R.; González Martín, A.; Cambra, O.; García Gil, O.; Odriozola, O.; López, O.; Escalante, S.; Lancharro, M. a Á. y López-Fraile, J. M. a (2016): "Pasados releídos: el dolmen del Portillo de las Cortes. Guadalajara”, Boletín del Museo Arqueológico Nacional, 34, pp. 9-28.

(C) Universidad de Salamanca
Bueno, P.; De Balbín, R. y Barroso, R. (2004): “Application d'une méthode d'analyse du territoire à partir de la situation des marqueurs graphiques à l'intérieur de la Péninsule Ibérique: le Tage International”, L'Anthropologie, 108, pp. 653-710.

Bueno, P.; De Balbín, R. y Barroso, R. (2008): "Models of integration of rock art and megalith builders in the International Tagus". En Bueno, P.; Barroso, R. y De Balbín, R. (eds.): Graphical markers y megalith builders in the International Tagus. Iberian Peninsula. BAR Intern. Ser., 1745. Oxford: Archaeopress, pp. 5-15.

Bueno, P.; De Balbín, R. y Barroso, R. (2009a): "Constructores de megalitos y marcadores gráficos. Diacronías y sincronías en el Atlántico Ibérico”. En De Balbín, R.; Bueno, P.; González, R. y Del Arco, C. (eds.): Grabados rupestres de la fachada atlántica europea y africana. BAR Intern. Ser., 2043. Oxford: Archaeopress, pp. 149-172.

Bueno, P.; González, R. y Del Arco, C. (2009b): "Análisis de las grafías megalíticas de Antequera y su entorno". En Dólmenes de Antequera. Tutela y valorización hoy. Cuadernos PH. Sevilla: Instituto Andaluz de Patrimonio, pp. 186-197.

Bueno, P.; De Balbín, R.; Díaz-Andreu, M. y AldeCOA, A. (1998): "Espacio habitacional/espacio gráfico: grabados al aire libre en el término de La Hinojosa (Cuenca)", Trabajos de Prehistoria, 55 (1), pp. 101-120.

Bueno, P.; Jiménez, P. y Barroso, R. (1995): "Prehistoria Reciente en el Noroeste de Guadalajara". En De Balbín, R.; Valiente, J. y Mussat, M. (eds.): Arqueología en Guadalajara. Guadalajara: AACHE edics., pp. 73-95.

Cerrillo, E. (2006): Los Barruecos: primeros resultados sobre el poblamiento neolitico de la cuenca extremeña del Tajo. Memorias de Arqueología Extremeña, 6. Mérida: Junta de Extremadura-Dirección General de Patrimonio Cultural. 
Cerrillo, E. (2011): "Planteamientos y nuevos datos para la interpretación de los paisajes prehistóricos del sector extremeño del Tajo: el área de Alconétar", Zephyrus, LXviII, pp. 139-161.

Cruz, M. (2004): "La investigación del arte rupestre desde la Geografía: la pintura neolítica del ámbito mediterráneo de la Península Ibérica”, Trabajos de Prehistoria, 61 (2), pp. 41-62.

FAIrÉN, S. (2002): "Visibilidad y percepción del entorno. Análisis de distribución del arte rupestre esquemático mediante sistemas de información geográfica”, Lucentum, 21-22, pp. 27-43.

FAIrÉn, S. (2004): “Arte, estilo y territorio. La construcción de un paisaje neolítico en las comarcas centro-meridionales valencianas”, Zephyrus, LVII, pp. 167-182.

Fisher, P.; Farrely, C.; Maddocks, A. y Ruggles, C. (1997): "Spatial analysis of visible areas from the Bronze Age cairns of Mull", Journal of Archaeological Science, 24, pp. 581-592.

Gaffney, V. y StÂncic, Z. (1991): GIS Approaches to Regional Analysis: a Case Study of the Island of Hvar. Znanstveni Institut Filozofske fakultete, Ljubljana.

García Sanjuán, L.; Metcalfe-Wood, S.; Rivera, T. y Wheatley, D. (2006): “Análisis de pautas de visibilidad en la distribución de monumentos megalíticos de Sierra Morena Occidental”. En Grau, I. (ed.): La aplicación de los SIG en la arqueología del paisaje. Serie Arqueológica. Alicante: Univ. de Alicante, pp. 181-200.

Gibson, E. (2007): “The Archaeology of Movement in a Mediterranean Landscape", Journal of Mediterranean Archaeology, 20 (1), pp. 61-87.

Gillings, M. (2015): "Mapping invisibility: GIS approaches to the analysis of hiding and seclusion", Journal of Archaeological Science, 62, pp. 1-14.

Guillem, P. y Martínez Valle, R. (2013): "Arte rupestre en el abric del Castell de Vilafamés (Castellón)". En Martínez García, J. y Hernández, M. (eds.): iI Congreso de Arte Rupestre Esquemático en la Península Ibérica (Comarca de los Vélez). Almería, pp. 203-213.

Higuchi, T. (1983): The visual and Spatial Structure of Landscapes. Massachusetts: Institute of Technology.

Lake, M. y WoOdman, P. (2003): "Visibility studies in archaeology: a review and case study", Environment and Planning B: Planning and Design, 30 (5), pp. 689-707.

Lake, M. W.; Woodman, P. E. y Mithen, S. J. (1998): "Tailoring GIs software for archaeological applications: An example concerning viewshed analysis", Journal of Archaeological Science, 25, pp. 27-38.
LANCharro, M.a Á. (2012): "Grafías y territorios de la Prehistoria Reciente en la cuenca interior del Tajo: Madrid y Toledo". En 1. a Mesa-Redonda Artes rupestres da Pré-História e da Proto-História: Paradigmas e metodologías de registo. Trabalhos de Arqueologia, 54, pp. 273-282.

Llobera, M. (2003): "Extending Gis-based visual analysis: the concept of visualscapes", International Journal of Geographical Information Science, 17 (1), pp. 25-48.

Llobera, M.; Wheatley, D.; Steele, J.; Cox, S. y Parchment, O. (2010): "Calculating the inherent visual structure of a landscape (inherent viewshed) using high-throughput computing". En NiccoLucci, F. y Hermon, S. (eds.): Beyond the artefact: Digital Interpretation of the Past: Proceedings of CAA2004 (Prato, 2004). Budapest: Archaeolingua, pp. 146-151.

Martínez Bea, M. (2006): “Arte rupestre y sig en los alrededores de Santolea (Teruel)”. En Grau, I. (ed.): La aplicación de los SIG en Arqueología. Alicante: Univ. de Alicante, pp. 171-180.

Martínez García, J. (1998): “Abrigos y accidentes geográficos como categorías de análisis en el paisaje de la pintura rupestre esquemática. El sudeste como marco", Arqueología Espacial, 19, pp. 543-562.

Martínez García, J. (2006): "La pintura rupestre esquemática en el proceso de consolidación de las sociedades productoras". En Martínez García, J. y Hernández Pérez, M. (eds.): Actas del Congreso de Arte Rupestre Esquemático en la Peninsula Ibérica (Comarca de los Vélez). Almería, pp. 33-56.

Martínez i Rubio, T. y Martorell, X. (2013): “La senda heredada: contribución al estudio de la red de caminos óptimos entre yacimientos de hábitat y de arte rupestre neolíticos en el Macizo del Caroig (Valencia)", Zephyrus, Lxx, pp. 69-84.

Murrieta, P. (2012): "Understanding human movement through spatial technologies. The role of natural areas of transit in the late Prehistory of South-western Iberia", Trabajos de Prehistoria, 69 (1), pp. 103-122.

Ogburn, D. (2006): "Assessing the level of visibility of cultural objects in past landscapes", Journal of Archaeological Science, 33, pp. 405-413.

Oliver, D.; Sagardoy, T.; Moreno, D. y Bravo, F. (2015): "Arte rupestre postpaleolítico en la provincia de Guadalajara: el abrigo de Los Forestales". En 1915-2015, 100 Anys Real Academia de Cultura Valénciana. Serie Arqueológica, 24. Varia, XII. Valencia, pp. 565-608. 
Parcero, C. (2002): La construcción del paisaje social en la Edad del Hierro en el Noroeste ibérico. Ortegalia 01. Ortigueira.

Ruiz, J. F.; Hernanz, A.; Armitage, R. A.; Rowe, M. W.; Viñas, R.; Gavira, J. M. ${ }^{a}$ y Rubio, A. (2012): "Calcium oxalate AMS I4C dating and chronology of post-Palaeolithic rock paintings in the Iberian Peninsula. Two dates from Abrigo de los Oculados (Henarejos, Cuenca, Spain)", Journal of Archaeological Science, 39 (8), pp. 2655-2667.

SAUCEDA, M. ${ }^{\text {I. }}$ (2001): Pinturas y grabados rupestres esquemáticos del monumento natural de Los Barruecos. Malpartida de Cáceres. Memorias, 2. Mérida: Edit. Reg. de Extremadura.

Van Leusen, M. (1999): "Line-of sight and cost surface analysis using GIS”. En BARCeló, J. A.; BRIZ, I. y VILA, A. (eds.): New Techniques for Old Times: Computer Applications in Archaeology. Oxford, pp. 215-223.

Wheatley, D. (1995): "Cumulative viewshed analysis: A grs-based method for investigating intervisibility, and its archaeological application". En Lock, G. y STÂNCIC, Z. (eds.): Archaeology and Geographical Information Systems. London: Taylor y Francis, pp. 171-186.

WheAtley, D. (1996): "The use of GIS to understand regional variation in earlier Neolithic Wessex". En Maschner, H. D. G. (ed.): New Methods, Old Problems: Geographic Information Systems, in Modern Archaeological Research. Occasional Paper, 23. Carbondale: Southern Illinois University Center for Archaeological Investigations, pp. 75-103.
Wheatley, D. (2014): “Connecting landscapes with built environments: visibility analysis, scale and the senses". En Paliou, E.; Lieberwirth, U. y Polla, S. (eds.): Spatial analysis and social spaces: interdisciplinary approaches to the interpretation of prehistoric and historic built environments. Berlin: De Gruyter, pp. 115-134.

Wheatley, D. y Gillings, M. (2000): "Vision, Perception and Gis: some notes on the development of enriched approaches to the study of archaeological visibility". En Lock, G. R. (ed.): Beyond the map: archaeology and spatial technologies. NATO ASI SERIES A LIFE SCIENCES, 321. Oxford: Institute of Archaeology, pp. 1-27.

Wheatley, D. y Gillings, M. (2002): Spatial technology and archaeology. The Archaeological Applications of GIS. New York.

Zamora, M. (2006): "Visibilidad y Arqueología, mucho más que ceros y unos". En Grau, I. (ed.): La aplicación de los SIG en la Arqueología del Paisaje. Alicante: Univ. de Alicante, pp. 41-54.

\section{Otras fuentes}

Carta arqueológica de Hombrados (CCAA 07/p o c 19/ municipio 139 y 165):

- yacimientos 02 y 03; redactor Arenas, J. A. (1999).

- yacimiento 547; redactores AlCOLEA, J. J. y JimÉNEZ, P. J. (1994).

— yacimiento 07 y 08; redactores Gómez, G. y GonzáLEZ, C. (2004). 